PROCEEDINGS OF THE

AMERICAN MATHEMATICAL SOCIETY

Volume 139, Number 3, March 2011, Pages 957-967

S 0002-9939(2010)10508-7

Article electronically published on July 28,2010

\title{
$p$-CONVERGENT SEQUENCES AND BANACH SPACES IN WHICH $p$-COMPACT SETS ARE $q$-COMPACT
}

\author{
CÁNDIDO PIÑEIRO AND JUAN MANUEL DELGADO
}

(Communicated by Nigel J. Kalton)

\begin{abstract}
We introduce and investigate the notion of $p$-convergence in a Banach space. Among others, a Grothendieck-like result is obtained; namely, a subset of a Banach space is relatively $p$-compact if and only if it is contained in the closed convex hull of a $p$-null sequence. We give a description of the topological dual of the space of all $p$-null sequences which is used to characterize the Banach spaces enjoying the property that every relatively $p$-compact subset is relatively $q$-compact $(1 \leq q<p)$. As an application, Banach spaces satisfying that every relatively $p$-compact set lies inside the range of a vector measure of bounded variation are characterized.
\end{abstract}

\section{INTRODUCTION}

By a well known characterization due to Grothendieck 7] (see, e.g., 9, p. 30]), a subset $A$ of a Banach space $X$ is relatively compact if and only if there exists $\left(x_{n}\right)$ in $c_{0}(X)$ (the space of norm-null sequences in $X$ ) such that $A \subset\left\{\sum_{n} a_{n} x_{n}: \sum_{n}\left|a_{n}\right| \leq\right.$ $1\}$. Since then, several authors have dealt with stronger forms of compactness, studying sets sitting inside the convex hulls of special types of null sequences. For instance, it was observed in [14] (see also [1]) that if one considers, instead of $c_{0}(X)$, the space of $q$-summable sequences $\ell_{q}(X)$, for some fixed $q \geq 1$, then this stronger form of compactness characterizes Reinov's approximation property of order $p$, $0<p<1$. This latter form of compactness was recently further strengthened by Sinha and Karn [15] as follows. Let $1 \leq p \leq \infty$ and let $p^{\prime}$ be the conjugate index of $p$ (i.e., $1 / p+1 / p^{\prime}=1$ ). The $p$-convex hull of a sequence $\left(x_{n}\right) \in \ell_{p}(X)$ is defined as $p$ - $\operatorname{co}\left(x_{n}\right)=\left\{\sum_{n} a_{n} x_{n}: \sum_{n}\left|a_{n}\right|^{p^{\prime}} \leq 1\right\}\left(\sup \left|a_{n}\right| \leq 1\right.$ if $\left.p=1\right)$. A set $A \subset X$ is said to be relatively $p$-compact if there exists $\left(x_{n}\right) \in \ell_{p}(X)\left(\left(x_{n}\right) \in c_{0}(X)\right.$ if $p=\infty$ ) such that $A \subset p$ - $\operatorname{co}\left(x_{n}\right)$. (Note that similar notions with $\left(x_{n}\right)$ being a weakly $p$-summable sequence were already considered in [2, p. 51].) Some results concerning this type of relatively compact set have been set in [3].

The aim of this article is to deepen the study of the geometry of Banach spaces related to $p$-compact sets. In this way, the notion of $p$-convergent sequence is introduced in Section 2 and a Grothendieck-like result is obtained; namely, a subset

Received by the editors January 21, 2010 and, in revised form, March 6, 2010 and March 22, 2010

2010 Mathematics Subject Classification. Primary 46B50, 47B07; Secondary 47B10.

Key words and phrases. $p$-compact set, $p$-convergent sequence, $p$-nuclear operator, $p$-summing operator, cotype.

This research was supported by MTM2009-14483-C02-01 project (Spain).

(C)2010 American Mathematical Society 
of a Banach space is relatively $p$-compact if and only if it is contained in the closed convex hull of a sequence $p$-convergent to zero (Theorem 2.5).

In [3, Theorem 3.14], Serrano and the authors have proved that every infinite dimensional Banach space has relatively compact sets failing to be $q$-compact for every $1 \leq q<\infty$. Section 3 is devoted to find out if this result is also true when we replace compact $(=\infty$-compact) with $p$-compact, $p>q$. In fact, we come to the same conclusion if $p>2$ (Proposition 3.5): if $1<q<p \leq 2$, it is shown that every relatively $p$-compact subset of $X$ is relatively $q$-compact if and only if every $q^{\prime}$ summing operator from $c_{0}$ to $X^{*}$ is $p^{\prime}$-summing $\left(p^{\prime}\right.$ and $q^{\prime}$ are conjugate exponents of $p$ and $q$, respectively). It is convenient to point out that the description of the topological dual of the space of the $p$-null sequences (Proposition 3.1) simplifies the proofs of this section.

Our notation is standard. A Banach space $X$ will be regarded as a subspace of its bidual $X^{* *}$ under the canonical embedding $i_{X}: X \rightarrow X^{* *}$. We denote the closed unit ball of $X$ by $B_{X}$. For Banach spaces $X$ and $Y$, the space of all bounded linear operators from $X$ to $Y$ is denoted by $\mathcal{L}(X, Y)$. We also need the following operator ideals: $\mathcal{N}_{p}-p$-nuclear operators, $\mathcal{Q N}_{p}-$ quasi $p$-nuclear operators, $\mathcal{I}_{p}-$ $p$-integral operators and $\Pi_{p}-p$-summing operators. We refer to Pietsch's book [11] for operator ideals (see also 5 , by Diestel, Jarchow and Tonge for common operator ideals $\mathcal{N}_{p}$ and $\Pi_{p}$, and [10] by Persson and Pietsch for $\mathcal{Q} \mathcal{N}_{p}$ ).

Let $1 \leq p \leq \infty$. The space of all weakly $p$-summable sequences (respectively, (strongly) $p$-summable sequences) in $X$ is denoted by $\ell_{p}^{w}(X)$ (respectively, $\ell_{p}(X)$ ) endowed with its natural norm $\left\|\left(x_{n}\right)\right\|_{p}^{w}$ (respectively, $\left.\left\|\left(x_{n}\right)\right\|_{p}\right)$. We write $\ell_{\infty}(X)$ to describe the space of all bounded sequences $\left(x_{n}\right)$ in $X$ with the norm $\left\|\left(x_{n}\right)\right\|_{\infty}$.

Relying on the notion of $p$-compactness, the notion of $p$-compact operator is defined in an obvious way (see [15]): an operator $T \in \mathcal{L}(X, Y)$ is said to be $p$ compact if $T\left(B_{X}\right)$ is relatively $p$-compact in $Y$. The class of all $p$-compact operators between Banach spaces is denoted by $\mathcal{K}_{p}$. It is shown in [15] that $\mathcal{K}_{p}$ is an operator ideal. Even more, $\mathcal{K}_{p}$, equipped with the norm $k_{p}$ defined by

$$
k_{p}(T)=\inf \left\{\left\|\left(y_{n}\right)\right\|_{p}:\left(y_{n}\right) \in \ell_{p}(Y) \text { and } T\left(B_{X}\right) \subset p-\operatorname{co}\left(y_{n}\right)\right\}
$$

for all $T \in \mathcal{K}_{p}(X, Y)$, is a Banach operator ideal. In order to make the article self-contained, we list some properties related to $p$-compactness:

- If $1 \leq q \leq p \leq \infty$, then every relatively $q$-compact set is $p$-compact.

- An operator $T: X \longrightarrow Y$ belongs to $\mathcal{K}_{p}(X, Y)$ (respectively, $\mathcal{Q N}_{p}(X, Y)$ ) if and only if $T^{*}$ belongs to $\mathcal{Q N}_{p}\left(Y^{*}, X^{*}\right)$ (respectively, $\mathcal{K}_{p}\left(Y^{*}, X^{*}\right)$ ) [3, Corollary 3.4 and Proposition 3.8].

- A sequence $\hat{x}=\left(x_{n}\right)$ of $X$ is relatively $p$-compact if and only if $U_{\hat{x}}: e_{n} \in$ $\ell_{1} \longmapsto x_{n} \in X$ is $p$-compact, where $\left(e_{n}\right)$ denotes the unit vector basis of $\ell_{1}$ [3, Proposition 3.5].

\section{2. $p$-CONVERGENCE}

Definition 2.1. Let $p \geq 1$. A sequence $\left(x_{n}\right)$ in a Banach space $X$ is said to be $p$-null if, for every $\varepsilon>0$, there exist $n_{0} \in \mathbb{N}$ and $\left(z_{k}\right) \in \varepsilon B_{\ell_{p}(X)}$ such that $x_{n} \in p$ - $\operatorname{co}\left(z_{k}\right)$ for all $n \geq n_{0}$.

A sequence $\left(x_{n}\right)$ in $X$ is said to be $p$-convergent if there exists $x \in X$ such that $\left(x_{n}-x\right)$ is $p$-null. 
Remark 2.2. Since every $p$-null sequence $\hat{x}=\left(x_{n}\right)$ has relatively $p$-compact rank, it induces an operator $U_{\hat{x}}: \ell_{1} \longrightarrow X$ defined by $U_{\hat{x}}\left(\alpha_{n}\right)=\sum_{n} \alpha_{n} x_{n}$ that is $p$ compact. It is easy to show that a bounded sequence $\hat{x}=\left(x_{n}\right)$ is $p$-null if and only if $U_{\hat{x}}=k_{p^{-}} \lim _{n} U_{\hat{x}(n)}$, where $\hat{x}(n)=\left(x_{1}, \ldots, x_{n}, 0, \ldots\right)$.

Remark 2.3. It is clear that $p$-summable sequences are $p$-null. For an example of a $p$-null sequence failing to be $p$-summable, consider $\left(n^{-1 / p} e_{n}\right)$ (where $\left(e_{n}\right)$ denotes the unit vector basis in $\ell_{2}$ ) which is a null sequence in $\ell_{2}$. From the next proposition, that sequence is $p$-null in $c_{0}$. Nevertheless, it does not belong to $\ell_{p}\left(c_{0}\right)$.

In [3, Theorem 3.14], it is proved that an operator $T \in \mathcal{L}(X, Y)$ has $p$-summing adjoint if and only if $T$ maps relatively compact sets in $X$ to relatively $p$-compacts sets in $Y$. The notion of $p$-null sequence allows us to establish the sequential version of this result:

Proposition 2.4. Let $p \geq 1$. An operator $T \in \mathcal{L}(X, Y)$ has $p$-summing adjoint if and only if $T$ maps null sequences in $X$ to $p$-null sequences in $Y$.

Proof. Suppose that $T^{*}$ is $p$-summing and let $\hat{x}=\left(x_{n}\right)$ be a null sequence in $X$. Notice that $U_{T \hat{x}}-U_{T \hat{x}(n)}=T \circ\left(U_{\hat{x}}-U_{\hat{x}(n)}\right)$ for each $n \in \mathbb{N}$. Now, the dual version of [3, Theorem 3.13] ensures that $T \circ\left(U_{\hat{x}}-U_{\hat{x}(n)}\right)$ belongs to $\mathcal{K}_{p}\left(\ell_{1}, Y\right)$ and

$$
k_{p}\left(T \circ\left(U_{\hat{x}}-U_{\hat{x}(n)}\right)\right) \leq \pi_{p}\left(T^{*}\right)\left\|U_{\hat{x}}-U_{\hat{x}(n)}\right\| .
$$

Since $\left\|U_{\hat{x}}-U_{\hat{x}(n)}\right\| \stackrel{n}{\longrightarrow} 0$, it follows that $U_{T \hat{x}}=k_{p}-\lim _{n} U_{T \hat{x}(n)}$.

The converse is obvious in view of [3, Theorem 3.14].

We denote by $c_{0, p}(X)$ the vector space of all $p$-null sequences in $X$ endowed with the norm $k_{p}(\hat{x})=k_{p}\left(U_{\hat{x}}\right)$. This space can be identified with a closed subspace of $\mathcal{K}_{p}\left(\ell_{1}, X\right)$ using a standard argument.

The following result is intended to make clear the analogy between the $p$-convergence and the convergence in a Banach space related to compactness.

Theorem 2.5. Let $p \geq 1$. A set in a Banach space $X$ is relatively $p$-compact if and only if it is contained in the closed convex hull of a p-null sequence.

Proof. If $A$ is relatively $p$-compact in $X$, there exists a sequence $\hat{z}=\left(z_{n}\right) \in \ell_{p}(X)$ so that $A \subset p$ - $\operatorname{co}\left(z_{n}\right)$. The sequence $\left(z_{n}\right)$ induces an operator $\phi_{\hat{z}}: e_{n} \in \ell_{p^{\prime}} \longmapsto$ $z_{n} \in X$ satisfying $\phi_{\hat{z}}\left(B_{\ell_{p^{\prime}}}\right)=p$ - $\operatorname{co}\left(z_{n}\right)$. In order to obtain a factorization of $\phi_{\hat{z}}$, choose $\left(\alpha_{n}\right) \searrow 0$ such that $\left(\alpha_{n}^{-1} z_{n}\right) \in \ell_{p}(X)$. Now, consider the compact operator $D: \ell_{p^{\prime}} \longrightarrow \ell_{p^{\prime}}$ and the $p$-compact operator $\phi: \ell_{p^{\prime}} \longrightarrow X$ defined by $D\left(\beta_{n}\right)=\left(\alpha_{n} \beta_{n}\right)$ and $\phi\left(\beta_{n}\right)=\sum_{n} \beta_{n} \alpha_{n}^{-1} x_{n}$. It is obvious that the following diagram is commutative:

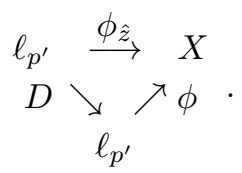

As $D\left(B_{\ell_{p^{\prime}}}\right)$ is relatively compact in $\ell_{p^{\prime}}$, we can find a null sequence $\left(\gamma_{n}\right)$ in $\ell_{p^{\prime}}$ with $D\left(B_{\ell_{p^{\prime}}}\right) \subset \overline{\mathrm{co}}\left(\gamma_{n}\right)$. Then

$$
A \subset \phi_{\hat{z}}\left(B_{\ell_{p^{\prime}}}\right)=\phi\left(D\left(B_{\ell_{p^{\prime}}}\right)\right) \subset \phi\left(\overline{\mathrm{co}}\left(\gamma_{n}\right)\right) \subset \overline{\mathrm{co}}\left(\phi\left(\gamma_{n}\right)\right) .
$$

Since $\phi$ is $p$-compact, $\phi^{*}$ is quasi $p$-nuclear [3, Corollary 3.4] and, in particular, $p$-summing. So, according to Proposition 2.4, the sequence $\left(\phi\left(\gamma_{n}\right)\right)$ is $p$-null in $X$. 
For the converse, just notice that the closed convex hull of relatively $p$-compact sets are $p$-compact.

We finish this section with a characterization of $p$-null sequences in Banach spaces having the $k_{p^{-}}$approximation property. A Banach space $X$ has the $k_{p^{-}}$ approximation property if, for every Banach space $Y$, the space $\mathcal{F}(Y, X)$ of all finite rank operators is $k_{p}$-dense in $\mathcal{K}_{p}(Y, X)$ [4. This property might be considered as a gradation of the classic approximation property. Since the classic approximation property implies the $k_{p}$-approximation property for all $p \geq 1$, the following result can be applied to a wide class of Banach spaces.

Proposition 2.6. If $X$ has the $k_{p}$-approximation property, then $\hat{x}=\left(x_{n}\right) \in c_{0, p}(X)$ if and only if $\hat{x} \in c_{0}(X)$ and has relatively p-compact rank.

Proof. Only the "if" part needs to be proved (the converse is straightforward from the definition of $p$-null sequences). So, given $\varepsilon>0$ and a sequence $\left(x_{n}\right) \in c_{0}(X)$ with relatively $p$-compact rank, there exists a finite rank operator $S: X \longrightarrow X$ such that $k_{p}\left(U_{\hat{x}}-S \circ U_{\hat{x}}\right)<\varepsilon / 3$ [4, Theorem 2.1]. According to Proposition 2.4, the sequence $S \hat{x}=\left(S x_{n}\right)$ is $p$-null and this allows us to find $n_{0} \in \mathbb{N}$ for which $k_{p}\left(U_{S \hat{x}}-U_{S \hat{x}(n)}\right)<\varepsilon / 3$ whenever $n \geq n_{0}$. Finally, if $P_{n}: \ell_{1} \longrightarrow \ell_{1}$ denotes the projection onto the first $n$-th coordinates, we have, for every $n \geq n_{0}$,

$$
\begin{aligned}
k_{p}\left(U_{\hat{x}}-U_{\hat{x}(n)}\right) & \leq k_{p}\left(U_{\hat{x}}-S \circ U_{\hat{x}}\right)+k_{p}\left(S \circ U_{\hat{x}}-S \circ U_{\hat{x}(n)}\right)+k_{p}\left(S \circ U_{\hat{x}(n)}-U_{\hat{x}(n)}\right) \\
& \leq k_{p}\left(U_{\hat{x}}-S \circ U_{\hat{x}}\right)+k_{p}\left(U_{S \hat{x}}-U_{S \hat{x}(n)}\right)+k_{p}\left(S \circ U_{\hat{x}}-U_{\hat{x}}\right)\left\|P_{n}\right\| \\
& <\varepsilon .
\end{aligned}
$$

In [4, it is shown that every Banach space has the $k_{2}$-approximation property. Nevertheless, we do not know whether the hypothesis of the $k_{p}$-approximation property can be omitted in the previous result for $p \neq 2$.

$$
\text { 3. On the Equality } \Pi_{p}\left(c_{0}, X^{*}\right)=\Pi_{q}\left(c_{0}, X^{*}\right)
$$

In [3], it is proved that, for every $q \geq 1$ and every infinite dimensional Banach space, there exist relatively compact subsets that are not relatively $q$-compact 3 , Theorem 3.14]. The objective of this section is to find out if this result is also true when we replace compact ( $=\infty$-compact) with $p$-compact, $p>q$. We begin with a description of the dual space $c_{0, p}(X)^{*}$ which will be very useful in several proofs. Recall that the trace functional (denoted by tr) is well defined and continuous on $\mathcal{N}_{1}\left(X, X^{* *}\right)$ if and only if $X^{*}$ has the approximation property.

Proposition 3.1. Let $X$ be a Banach space and $p \geq 1$. The dual space $c_{0, p}(X)^{*}$ is isometrically isomorphic to $\Pi_{p^{\prime}}\left(c_{0}, X^{*}\right)$.

Proof. Given $S \in \Pi_{p^{\prime}}\left(c_{0}, X^{*}\right)$, we consider the linear form $f_{S}$ on $c_{0, p}(X)$ defined by $f_{S}(\hat{x})=\operatorname{tr}\left(U_{\hat{x}}^{*} \circ S\right)$. Since $U_{\hat{x}}$ is $p$-compact, the adjoint $U_{\hat{x}}^{*}: X^{*} \longrightarrow \ell_{\infty}$ is quasi $p$-nuclear [3, Corollary 3.4]; in particular, $U_{\hat{x}}^{*}$ is $p$-summing and the following inequalities hold:

$$
\pi_{p}\left(U_{\hat{x}}^{*}\right) \leq \nu_{p}^{Q}\left(U_{\hat{x}}^{*}\right) \leq k_{p}\left(U_{\hat{x}}\right) .
$$

Then, the composition $U_{\hat{x}}^{*} \circ S \in \Pi_{1}\left(c_{0}, \ell_{\infty}\right)=\mathcal{N}_{1}\left(c_{0}, \ell_{\infty}\right)$ and $\nu_{1}\left(U_{\hat{x}}^{*} \circ S\right) \leq$ $\pi_{p}\left(U_{\hat{x}}^{*}\right) \pi_{p^{\prime}}(S)$ [17, p. 55]. Since $\ell_{1}$ has the approximation property, the map $f_{S}$ is well defined and

$$
\left|\operatorname{tr}\left(U_{\hat{x}}^{*} \circ S\right)\right| \leq \nu_{1}\left(U_{\hat{x}}^{*} \circ S\right) \leq k_{p}\left(U_{\hat{x}}\right) \pi_{p^{\prime}}(S) .
$$


Therefore, $f_{S} \in c_{0, p}(X)^{*}$ and $\left\|f_{S}\right\| \leq \pi_{p^{\prime}}(S)$.

On the other hand, if $f \in c_{0, p}(X)^{*}$, we are able to define the operator $S_{f}: \alpha \in$ $c_{0} \longmapsto S_{f} \alpha \in X^{*}$ so that $\left\langle S_{f} \alpha, x\right\rangle=f(\alpha \otimes x)$ for all $x \in X$, where $\alpha \otimes x:=\left(\alpha_{n} x\right)$ if $\alpha=\left(\alpha_{n}\right)$. To see that $S_{f} \in \Pi_{p^{\prime}}\left(c_{0}, X^{*}\right)$, consider $\left(\alpha_{k}\right) \in \ell_{p^{\prime}}^{w}\left(c_{0}\right)$ with $\left.\|\left(\alpha_{k}\right)\right) \|_{p^{\prime}}^{w} \leq 1$ and let us prove

$$
\left(\sum_{k}\left\|S_{f} \alpha_{k}\right\|^{p^{\prime}}\right)^{1 / p^{\prime}} \leq\|f\|
$$

Fix $N \in \mathbb{N}, x_{1}, \ldots, x_{N} \in B_{X}$ and $\left(\beta_{k}\right)_{k=1}^{N} \in B_{\ell_{p}^{N}}$. We have

$$
\left|\sum_{k=1}^{N}\left\langle S_{f} \alpha_{k}, x_{k}\right\rangle \beta_{k}\right|=\left|f\left(\sum_{k=1}^{N} \alpha_{k} \otimes \beta_{k} x_{k}\right)\right| \leq\|f\| k_{p}\left(\sum_{k=1}^{N} \alpha_{k} \otimes \beta_{k} x_{k}\right) .
$$

Set $\hat{y}=\sum_{k=1}^{N} \alpha_{k} \otimes \beta_{k} x_{k}$. Notice that $\left(\alpha_{k}^{n}\right)_{n} \in B_{\ell_{p^{\prime}}}$ for each $k \in \mathbb{N}$, so we can ensure that $U_{\hat{y}} e_{n}^{*}=\sum_{k=1}^{N} \alpha_{k}^{n} \beta_{k} x_{k}$ belongs to the closed and absolutely convex set $p$ - $\operatorname{co}\left(\beta_{k} x_{k}\right)_{k=1}^{N}$ for each $n \in \mathbb{N}$ (here, $\left(e_{n}^{*}\right)$ denotes the unit vector basis of $\left.\ell_{1}\right)$. From this, it is clear that

$$
k_{p}(\hat{y})=k_{p}\left(U_{\hat{y}}\right) \leq\left\|\left(\beta_{k} x_{k}\right)_{k=1}^{N}\right\|_{p} \leq 1
$$

Summing up,

$$
\left|\sum_{k=1}^{N}\left\langle S_{f} \alpha_{k}, x_{k}\right\rangle \beta_{k}\right| \leq\|f\|
$$

for all $N \in \mathbb{N}, x_{1}, \ldots, x_{N} \in B_{X}$ and $\left(\beta_{k}\right)_{k=1}^{N} \in B_{\ell_{p}^{N}}$, from which (3.1) is obtained, using a standard argument. So $S_{f}$ is $p^{\prime}$-summing and, moreover, $\pi_{p^{\prime}}(S) \leq\|f\|$.

Now, the functionals $f_{S_{f}}$ and $f$ coincide on finitely supported sequences in $c_{0, p}(X)$. Indeed, no matter how we choose $N \in \mathbb{N}$ and $\hat{x}_{0}=\left(x_{1}, \ldots, x_{N}, 0,0, \ldots\right)$,

$$
\begin{aligned}
f\left(\hat{x}_{0}\right) & =\sum_{k=1}^{N} f\left(e_{k} \otimes x_{k}\right)=\sum_{k=1}^{N}\left\langle S_{f} e_{k}, U_{\hat{x}_{0}} e_{k}^{*}\right\rangle \\
& =\sum_{k=1}^{N}\left\langle\left(U_{\hat{x}_{0}}^{*} \circ S_{f}\right) e_{k}, e_{k}^{*}\right\rangle=\operatorname{tr}\left(U_{\hat{x}_{0}}^{*} \circ S_{f}\right)=f_{S}\left(\hat{x}_{0}\right) .
\end{aligned}
$$

Since $\hat{x}=k_{p}-\lim _{n} \hat{x}(n)$ for every $\hat{x} \in c_{0, p}(X)$ (Remark 2.2), it is clear that the functionals $f_{S_{f}}$ and $f$ coincide on $c_{0, p}(X)$.

The following result turns out to be the key to answer the question posed at the beginning of this section. As usual, if $X$ and $Y$ are Banach spaces and $x^{*} \in X^{*}$ and $y \in Y$ are fixed, $x^{*} \otimes y$ denotes the one dimensional operator from $X$ to $Y$ defined by $\left(x^{*} \otimes y\right) x=\left\langle x^{*}, x\right\rangle y$ for all $x \in X$.

Theorem 3.2. Let $X$ and $Y$ be Banach spaces and let $1 \leq q<p<\infty$. The following statements are equivalent for an operator $T \in \mathcal{L}(X, Y)$ :

a) The operator $T$ maps relatively $p$-compact subsets of $X$ to relatively $q$ compact subsets of $Y$.

b) The operator $T^{*} \circ u \in \Pi_{p^{\prime}}\left(c_{0}, X^{*}\right)$ whenever $u \in \Pi_{q^{\prime}}\left(c_{0}, Y^{*}\right)\left(u \in \mathcal{L}\left(c_{0}, Y^{*}\right)\right.$, in case $q=1$ ). 
Proof. $a) \Rightarrow b)$ It is easy to prove that the operator $V: u \in \mathcal{K}_{p}\left(\ell_{1}, X\right) \longmapsto T \circ u \in$ $\mathcal{K}_{q}\left(\ell_{1}, Y\right)$ has closed graph. Then there exists a positive constant $C$ such that

$$
k_{q}(T \circ u) \leq C k_{p}(u) \text { for all } u \in \mathcal{K}_{p}\left(\ell_{1}, X\right) .
$$

In view of this, for every $p$-null sequence $\hat{x}$ we have

$$
k_{q}\left(U_{T \hat{x}}-U_{T \hat{x}(n)}\right)=k_{q}\left(T \circ\left(U_{\hat{x}}-U_{\hat{x}(n)}\right)\right) \leq C k_{p}\left(U_{\hat{x}}-U_{\hat{x}(n)}\right) .
$$

So, from Remark 2.2. $T$ maps $p$-null sequences to $q$-null sequences and, therefore, we can consider the operator $\widehat{T}:\left(x_{n}\right) \in c_{0, p}(X) \rightarrow\left(T x_{n}\right) \in c_{0, q}(Y)$. It only remains to prove that the adjoint $\widehat{T}^{*}: \Pi_{q^{\prime}}\left(c_{0}, Y^{*}\right) \longrightarrow \Pi_{p^{\prime}}\left(c_{0}, X^{*}\right)$ is defined by $\widehat{T}^{*}(u)=$ $T^{*} \circ u$ for all $u \in \Pi_{q^{\prime}}\left(c_{0}, Y^{*}\right)$. To see this, consider $x \in X, \beta=\left(\beta_{n}\right) \in c_{0}$ and $u \in \Pi_{q^{\prime}}\left(c_{0}, Y^{*}\right)$ and denote the sequence $\left(\beta_{n} x\right)$ by $\beta \otimes x$. On one hand, we have

$$
\begin{aligned}
\left\langle\widehat{T}^{*}(u), \beta \otimes x\right\rangle & =\operatorname{tr}\left(U_{\beta \otimes x}^{*} \circ \widehat{T}^{*}(u)\right)=\operatorname{tr}\left((x \otimes \beta) \circ \widehat{T}^{*}(u)\right) \\
& =\operatorname{tr}\left(\left(\widehat{T}^{*}(u)^{*} x\right) \otimes \beta\right)=\left\langle\widehat{T}^{*}(u)^{*} x, \beta\right\rangle=\left\langle x, \widehat{T}^{*}(u) \beta\right\rangle .
\end{aligned}
$$

On the other hand,

$$
\begin{aligned}
\langle u, \widehat{T}(\beta \otimes x)\rangle & =\operatorname{tr}\left(U_{\beta \otimes T x}^{*} \circ u\right)=\operatorname{tr}((T x \otimes \beta) \circ u) \\
& =\operatorname{tr}\left(u^{*}(T x) \otimes \beta\right)=\left\langle u^{*}(T x), \beta\right\rangle=\left\langle x,\left(T^{*} \circ u\right) \beta\right\rangle .
\end{aligned}
$$

As $\left\langle\widehat{T}^{*}(u), \beta \otimes x\right\rangle=\langle u, \widehat{T}(\beta \otimes x)\rangle$, it follows that $\left\langle x, \widehat{T}^{*}(u) \beta\right\rangle=\left\langle x,\left(T^{*} \circ u\right) \beta\right\rangle$ for all $x \in X$ and $\beta \in c_{0}$, which yields $\widehat{T}^{*}(u)=T^{*} \circ u$.

$b) \Rightarrow a$ ) It is a standard argument to prove that the operator

$$
u \in \Pi_{q^{\prime}}\left(c_{0}, Y^{*}\right) \longmapsto T^{*} \circ u \in \Pi_{p^{\prime}}\left(c_{0}, X^{*}\right)
$$

has closed graph and, therefore, that it is continuous. If $\Phi$ denotes the restriction of this operator to $\mathcal{N}_{q^{\prime}}\left(c_{0}, Y^{*}\right)$, then $\Phi^{*}: c_{0, p}(X)^{* *} \longrightarrow \Pi_{q}\left(Y^{*}, \ell_{\infty}\right)$ is defined by $\Phi^{*}(\hat{x})=U_{\hat{x}}^{*} \circ T^{*}$ for all $\hat{x} \in c_{0, p}(X)$ (just using a similar argument as in $\left.a\right) \Rightarrow b$ ) above). Since $U_{\hat{x}}^{*} \circ T^{*}=U_{T \hat{x}}^{*}$, the restriction of $\Phi^{*}$ to $c_{0, p}(X)$ is the operator

$$
\hat{x} \in c_{0, p}(X) \longmapsto U_{T \hat{x}}^{*} \in \Pi_{q}\left(Y^{*}, \ell_{\infty}\right) .
$$

Now, we will show that $\Phi^{*}$ maps $c_{0, p}(X)$ to $\mathcal{Q N}_{q}\left(Y^{*}, \ell_{\infty}\right)$. According to [16, Theorem 2.6], the $q$-summing norm and the quasi $q$-nuclear norm of a finite rank operator coincide. So, given $\hat{x} \in c_{0, p}(X)$, we have for $m>n$ :

$\nu_{q}^{Q}\left(\Phi^{*}(\hat{x}(m))-\Phi^{*}(\hat{x}(n))\right)=\pi_{q}\left(\Phi^{*}(\hat{x}(m))-\Phi^{*}(\hat{x}(n))\right) \leq\left\|\Phi^{*}\right\| k_{p}(\hat{x}(m)-\hat{x}(n))$.

This proves that $\left(\Phi^{*}(\hat{x}(n))\right)$ is a Cauchy sequence in $\mathcal{Q N}_{q}\left(Y^{*}, \ell_{\infty}\right)$ from which it is easy to conclude that $\Phi^{*}(\hat{x}) \in \mathcal{Q N}_{q}\left(Y^{*}, \ell_{\infty}\right)$. Hence, we can replace $\Pi_{q}\left(Y^{*}, \ell_{\infty}\right)$ with $\mathcal{Q N}_{q}\left(Y^{*}, \ell_{\infty}\right)$ in (3.2). In view of [3, Proposition 3.5], this means that $T$ maps $p$-null sequences to sequences with relatively $q$-compact rank. The proof concludes by just invoking Theorem 2.5

Let $\mathcal{P}_{p, q}(1 \leq q<p)$ be the class consisting of all Banach spaces with the property that every relatively $p$-compact subset is relatively $q$-compact. 
Corollary 3.3. Let $1 \leq q<p<\infty$. The following statements are equivalent for a Banach space $X$ :

a) $X \in \mathcal{P}_{p, q}$.

b) $\Pi_{q^{\prime}}\left(c_{0}, X^{*}\right)=\Pi_{p^{\prime}}\left(c_{0}, X^{*}\right)\left(\mathcal{L}\left(c_{0}, X^{*}\right)=\Pi_{p^{\prime}}\left(c_{0}, X^{*}\right)\right.$ if $\left.q=1\right)$.

In [6], the class of Banach spaces $Y$ satisfying

$$
\Pi_{2}\left(c_{0}, Y\right)=\mathcal{L}\left(c_{0}, Y\right)
$$

is deeply studied. Having in mind that $\mathcal{L}\left(c_{0}, Y\right)=\Pi_{\infty}\left(c_{0}, Y\right)$, Corollary 3.3 reveals that a dual Banach space $Y=X^{*}$ enjoys (3.3) if and only if relatively 2-compact subsets of $X$ are relatively 1-compact. Related to the ideas of [6], several conditions equivalent to Corollary $3.3(b)$ are established in the following result, the proof of which is omitted since it is quite similar to the proof in [6, Proposition 2.1].

Proposition 3.4. Let $X$ be a Banach space and let $1 \leq r<s$. The following statements are equivalent:

a) $\Pi_{r}(Y, X)=\Pi_{s}(Y, X)$ for every $\mathcal{L}_{\infty}$-space $Y$.

b) $\Pi_{r}(Y, X)=\Pi_{s}(Y, X)$ for some infinite dimensional $\mathcal{L}_{\infty}$-space $Y$.

c) There exists a positive constant $C$ such that $\pi_{r}(u) \leq C \pi_{s}(u)$ for all $n \in \mathbb{N}$ and $u \in \mathcal{L}\left(\ell_{\infty}^{n}, X\right)$.

Proposition 3.5. Let $1 \leq q<p$ and let $X$ be a Banach space.

1) If $X \in \mathcal{P}_{p, q}$ and $E$ is a closed subspace of $X$, then $X / E \in \mathcal{P}_{p, q}$.

2) $X^{* *} \in \mathcal{P}_{p, q}$ if and only if $X \in \mathcal{P}_{p, q}$.

3) If $2 \leq q<p$, then $\mathcal{P}_{p, q}$ does not contain any infinite dimensional Banach space.

Proof. To prove 1), notice that $u \in \Pi_{q^{\prime}}\left(c_{0},(X / E)^{*}\right)=\Pi_{q^{\prime}}\left(c_{0}, E^{\perp}\right)$ if and only if $i_{E^{\perp}} \circ u \in \Pi_{q^{\prime}}\left(c_{0}, X^{*}\right)$, where $i_{E^{\perp}}$ denotes the inclusion map from $E^{\perp}$ into $X^{*}$. By hypothesis and using the injectivity of the ideal of $p^{\prime}$-summing operators, it follows that $u \in \Pi_{p^{\prime}}\left(c_{0},(X / E)^{*}\right)$.

If $X^{* *} \in \mathcal{P}_{p, q}$, then a similar argument allows us to obtain that $X \in \mathcal{P}_{p, q}$. The converse follows easily from Proposition 3.4(c) using the local reflexivity principle.

Let us argue by contradiction to show 3 ). Suppose there exists an infinite dimensional Banach space $X \in \mathcal{P}_{p, q}$. By virtue of Corollary 3.3. there exists a positive constant $C$ such that

$$
\pi_{p^{\prime}}(u) \leq C \pi_{q^{\prime}}(u)
$$

for all $u \in \Pi_{q^{\prime}}\left(c_{0}, X^{*}\right)$. Now, for every $\gamma=\left(\gamma_{n}\right) \in \ell_{q^{\prime}}$, consider the operator

$$
D_{\gamma}:\left(x_{n}^{*}\right) \in \ell_{q}^{w}\left(X^{*}\right) \longmapsto\left(\gamma_{n} x_{n}^{*}\right) \in \ell_{p^{\prime}}\left(X^{*}\right) .
$$

To see that $D_{\gamma}$ is well defined, take $\left(x_{n}^{*}\right) \in \ell_{q}^{w}\left(X^{*}\right)$ and define the operators $A: c_{0} \longrightarrow \ell_{q^{\prime}}$ and $B: \ell_{q^{\prime}} \longrightarrow X^{*}$ by $A\left(\alpha_{n}\right)=\left(\gamma_{n} \alpha_{n}\right)$ and $B\left(\beta_{n}\right)=\sum_{n} \beta_{n} x_{n}^{*}$. It is easy to prove that $A$ is $q^{\prime}$-summing with $\pi_{q^{\prime}}(A) \leq\|\gamma\|_{q^{\prime}}$ and $\|B\| \leq 1$. Hence, $B \circ A$ is $q^{\prime}$-summing and, by hypothesis, $p^{\prime}$-summing. Moreover, in view of (3.4), we have

$$
\begin{aligned}
\left(\sum_{n}\left\|\gamma_{n} x_{n}^{*}\right\|^{p^{\prime}}\right)^{1 / p^{\prime}} & =\left(\sum_{n}\left\|(B \circ A) e_{n}\right\|^{p^{\prime}}\right)^{1 / p^{\prime}} \\
& \leq \pi_{p^{\prime}}(B \circ A) \leq C \pi_{q^{\prime}}(B \circ A) \leq C\|\gamma\|_{q^{\prime}}\left\|\left(x_{n}^{*}\right)\right\|_{q}^{w} .
\end{aligned}
$$


This shows that $D_{\gamma}$ is well defined and continuous for all $\gamma \in \ell_{q^{\prime}}$. In particular, the inequality

$$
\left(\sum_{n=1}^{N}\left\|\gamma_{n} x_{n}^{*}\right\|^{p^{\prime}}\right)^{1 / p^{\prime}} \leq C\|\gamma\|_{q^{\prime}}\left\|\left(x_{n}^{*}\right)_{n=1}^{N}\right\|_{q}^{w}
$$

holds for all $N \in \mathbb{N}$ and $x_{1}^{*}, \ldots, x_{N}^{*} \in X^{*}$. According to Dvoretzky-Rogers' Theorem [11, p. 38], given $\varepsilon>0$, for every $N \in \mathbb{N}$ there exist unitary vectors $x_{1}^{*}, \ldots, x_{N}^{*} \in X^{*}$ such that $\left\|\left(x_{n}^{*}\right)_{n=1}^{N}\right\|_{2}^{w} \leq(1+\varepsilon)$. As $q \geq 2$, applying (3.5) to these vectors, it follows that

$$
\left(\sum_{n=1}^{N}\left|\gamma_{n}\right|^{p^{\prime}}\right)^{1 / p^{\prime}} \leq C\|\gamma\|_{q^{\prime}}(1+\varepsilon)
$$

for all $N \in \mathbb{N}$. This is a contradiction if we take $\gamma \in \ell_{q^{\prime}} \backslash \ell_{p^{\prime}}$.

If $1 \leq q<p \leq 2$, the following proposition shows that there are (infinite dimensional) spaces in which relatively $p$-compact sets are relatively $q$-compact.

Proposition 3.6. The following statements are equivalent for a Banach space $X$ :

a) $X^{*}$ has finite cotype.

b) There exist $p, q \in \mathbb{R}, 1 \leq q<p \leq 2$, such that $X \in \mathcal{P}_{p, q}$.

In addition, if $X^{*}$ has cotype $s>2$ (respectively, $s=2$ ), then $X \in \mathcal{P}_{p, q}$ for every $1 \leq q<p<s^{\prime}$ (respectively, $1 \leq q<p \leq 2$ ).

Proof. $a) \Rightarrow b$ ) Suppose $X^{*}$ has finite cotype $s>2$ (respectively, $s=2$ ). According to [5. Theorem 11.14], we have $\Pi_{r}\left(c_{0}, X^{*}\right)=\mathcal{L}\left(c_{0}, X^{*}\right)$ for all $r>s$ (respectively, $\left.\Pi_{2}\left(c_{0}, X^{*}\right)=\mathcal{L}\left(c_{0}, X^{*}\right)\right)$. So, by Corollary 3.3, $X$ belongs to $\mathcal{P}_{r^{\prime}, 1}$ (respectively, $X$ belongs to $\mathcal{P}_{2,1}$ ).

$b) \Rightarrow a$ ) By contradiction, if $X^{*}$ does not have finite cotype, then $X^{*}$ contains $\ell_{\infty}^{n}$ uniformly [5, Theorem 14.1]. Thus, there is a constant $\lambda>0$ such that, for all $n \in \mathbb{N}$, there exists an isomorphism $J_{n}$ from $\ell_{\infty}^{n}$ onto a finite dimensional subspace $E_{n}$ of $X^{*}$ satisfying

$$
\left\|J_{n}\right\|\left\|J_{n}^{-1}\right\| \leq \lambda \text { for all } n \in \mathbb{N} .
$$

Suppose that $p$ and $q>1$ are real numbers satisfying $b$ ) (if $q=1$, the proof is quite similar). A call to Corollary 3.3 tells us that there exists a constant $C>0$ such that

$$
\pi_{p^{\prime}}(u) \leq C \pi_{q^{\prime}}(u) \text { for all } u \in \mathcal{L}\left(\ell_{\infty}^{n}, X^{*}\right) \text { and } n \in \mathbb{N} .
$$

If $I_{\infty}^{n}$ denotes the identity map on $\ell_{\infty}^{n}$, (3.6) and (3.7) yield

$$
\pi_{p^{\prime}}\left(I_{\infty}^{n}\right) \leq\left\|J_{n}^{-1}\right\| \pi_{p^{\prime}}\left(J_{n}\right) \leq C\left\|J_{n}^{-1}\right\| \pi_{q^{\prime}}\left(J_{n}\right) \leq C \lambda \pi_{q^{\prime}}\left(I_{\infty}^{n}\right) .
$$

On one hand, we have the estimation

$$
n^{1 / p^{\prime}}=\left(\sum_{k=1}^{n}\left\|I_{\infty}^{n}\left(e_{k}\right)\right\|^{p^{\prime}}\right)^{1 / p^{\prime}} \leq \pi_{p^{\prime}}\left(I_{\infty}^{n}\right),
$$

where $\left(e_{n}\right)$ is the canonical vector basis in $\ell_{\infty}^{n}$. On the other hand, as $I_{\infty}^{n}$ admits the $q^{\prime}$-nuclear representation $I_{\infty}^{n}=\sum_{k=1}^{n} e_{k}^{*} \otimes e_{k}$, it follows that

$$
\nu_{q^{\prime}}\left(I_{\infty}^{n}\right) \leq\left\|\left(e_{k}^{*}\right)\right\|_{q^{\prime}}\left\|\left(e_{k}\right)\right\|_{q}^{w} \leq n^{1 / q^{\prime}} .
$$


Since $\pi_{q^{\prime}}\left(I_{\infty}^{n}\right)=\nu_{q^{\prime}}\left(I_{\infty}^{n}\right)$ [17, p. 49], (3.8), (3.9) and (3.10) lead us to conclude

$$
n^{1 / p^{\prime}} \leq \pi_{p^{\prime}}\left(I_{\infty}^{n}\right) \leq C \lambda \pi_{q^{\prime}}\left(I_{\infty}^{n}\right) \leq C \lambda n^{1 / q^{\prime}}
$$

for all $n \in \mathbb{N}$, which is a contradiction since $q^{\prime}>p^{\prime}$.

Remark 3.7. The additional information in the above result cannot be improved for spaces whose dual has cotype $s>2$. Indeed, if $s^{\prime} \leq p \leq 2$, let us show that $X=\ell_{s^{\prime}} \notin \mathcal{P}_{p, q}$ for all $q<p$. First, put $p=s^{\prime}$ and suppose, by contradiction, that there exists $q<s^{\prime}$ satisfying $\ell_{s^{\prime}} \in \mathcal{P}_{s^{\prime}, q}$. In view of Proposition 3.6. $\ell_{s^{\prime}}$ belongs to $\mathcal{P}_{s^{\prime}, 1}$; that is, $\mathcal{L}\left(\ell_{\infty}, \ell_{s}\right)=\Pi_{s}\left(\ell_{\infty}, \ell_{s}\right)$, which contradicts [8, Theorem 7]. Now, for the case $p>s^{\prime}$, it suffices to see that $X=\ell_{s^{\prime}} \notin \mathcal{P}_{p, q}$ when $q$ satisfies $s^{\prime} \leq q<p$. Under this assumption, consider a sequence $\left(\lambda_{n}\right) \in \ell_{p} \backslash \ell_{q}$. The rank of the sequence $\left(\lambda_{n} e_{n}\right)$ is obviously relatively $p$-compact in $X$. Arguing again by contradiction, if the sequence is relatively $q$-compact, then the operator $U: e_{n} \in \ell_{1} \longmapsto \lambda_{n} e_{n} \in X$ is $q$-compact. Therefore, $U^{*}$ is quasi $q$-nuclear [3, Corollary 3.4] and, in particular, $q$-summing. As $\left(e_{n}^{*}\right) \in \ell_{s^{\prime}}^{w}\left(X^{*}\right) \subset \ell_{q}^{w}\left(X^{*}\right)$, it follows that

$$
\sum_{n}\left|\lambda_{n}\right|^{q}=\sum_{n}\left\|U^{*} e_{n}^{*}\right\|_{\infty}^{q}<+\infty,
$$

which is a contradiction (here, $\left(e_{n}^{*}\right)$ designs the unit vector basis of $X^{*}=\ell_{s}$ ). Finally, notice that this latter fact can be extended to $\mathcal{L}_{s^{\prime}}$-spaces, since every infinite dimensional $\mathcal{L}_{r}$-space has a complemented subspace isomorphic to $\ell_{r}$.

Remark 3.8. In general, the classes $\mathcal{P}_{p, q}$ are not closed under closed subspaces. For an example, consider $X=\ell_{\infty} ; X^{*}$ has cotype 2 since it is an $\mathcal{L}_{1}$-space. Therefore, $\ell_{\infty}$ belongs to $\mathcal{P}_{2,1}$. Nevertheless, $\ell_{1}$ is isometrically isomorphic to a closed subspace of $\ell_{\infty}$ but $\ell_{1} \notin \mathcal{P}_{2,1}$ (Proposition 3.6).

We finish with an application of the preceding results to the theory of vector measures. It is well known that only finite dimensional Banach spaces $X$ have the property that every relatively compact subset of $X$ lies inside the range of a vector measure of bounded variation [13, Theorem 3.6]. We are going to prove that if we replace compact $(=\infty$-compact $)$ with $p$-compact $(p \leq 2)$, then there exist infinite dimensional Banach spaces with such a property. It suffices to deal with $p$-null sequences instead of relative $p$-compact sets (Theorem 2.5), so the following lemma gains importance for getting our objective:

Lemma ([12, Lemma 2]). Let $\hat{x}$ be a bounded sequence in a Banach space $X$.

1) The rank of $\hat{x}$ lies inside the range of a $X^{* *}$-valued measure of bounded variation if and only if the operator $U_{\hat{x}}: \ell_{1} \longrightarrow X$ is 1-integral.

2) If $U_{\hat{x}}$ is 1-nuclear, then the rank of $\hat{x}$ lies inside the range of an $X$-valued measure of bounded variation.

Proposition 3.9. Let $X$ be a Banach space and let $1 \leq p<+\infty$. Every relatively $p$-compact subset of $X$ lies inside the range of a vector measure of bounded variation if and only if $X$ belongs to $\mathcal{P}_{p, 1}$.

Proof. If every relatively $p$-compact subset of $X$ lies inside the range of a vector measure of bounded variation, the previous lemma guarantees that the operator $\Phi: \hat{x} \in c_{0, p}(X) \longmapsto U_{\hat{x}} \in \mathcal{I}_{1}\left(\ell_{1}, X\right)$ is well defined. A standard argument shows 
that $\Phi$ has closed graph and, therefore, is continuous. Actually, $\Phi$ maps $c_{0, p}(X)$ into $\mathcal{N}_{1}\left(\ell_{1}, X\right)$, since $\hat{x}=k_{p}-\lim _{n} \hat{x}(n)$ whenever $\hat{x} \in c_{0, p}(X)$ and using that $\mathcal{N}_{1}\left(\ell_{1}, X\right)$ is isometrically isomorphic to a closed subspace of $\mathcal{I}_{1}\left(\ell_{1}, X\right)$ [11, p. 132]. Obviously, this implies that every relatively $p$-compact subset of $X$ is relatively 1-compact.

For the converse, notice that the operator $U_{\hat{x}}$ belongs to $\mathcal{K}_{1}\left(\ell_{1}, X\right)$ whenever $\hat{x}$ is a relatively $p$-compact sequence in $X$. Since $\mathcal{K}_{1}\left(\ell_{1}, X\right)$ can be isometrically identified with $\mathcal{N}_{1}\left(\ell_{1}, X\right)$, the proof is concluded via [12, Lemma 2].

In view of this result and Proposition [3.6. for a fixed $p \in[1,2)$ (respectively, $p=2$ ), every Banach space $X$ such that $X^{*}$ has cotype $s>p^{\prime}$ (respectively, $s=2$ ) satisfies that its relatively $p$-compact sets lies in the range of an $X$-valued measure of bounded variation.

\section{ACKNOWLEDGEMENT}

The authors wish to thank the referee, whose suggestions enhanced the clarity of this paper.

\section{REFERENCES}

1. J. Bourgain and O. Reinov, On the approximation properties for the space $H^{\infty}$, Math. Nachr. 122 (1985), 19-27. MR871186 (88e:46022)

2. J. M. F. Castillo and F. Sanchez, Dunford-Pettis-like properties of continuous vector function spaces, Rev. Mat. Univ. Complut. Madrid 6 (1993), 43-59. MR.1245024 (95e:46045)

3. J. M. Delgado, C. Piñeiro and E. Serrano, Operators whose adjoints are quasi p-nuclear, Studia Math. 197 (2010), 291-304.

4. _ Density of finite rank operators in the Banach space of p-compact operators, J. Math. Anal. Appl. 370 (2010), 498-505.

5. J. Diestel, H. Jarchow and A. Tonge, Absolutely Summing Operators, Cambridge Univ. Press, Cambridge, 1995. MR1342297 (96i:46001)

6. E. Dubinsky, A. Pelczynski and H. P. Rosenthal, On Banach spaces $X$ for which $\Pi_{2}\left(\mathcal{L}_{\infty}, X\right)=$ $B\left(\mathcal{L}_{\infty}, X\right)$, Studia Math. 44 (1972), 617-648. MR0365097 (51:1350)

7. A. Grothendieck, Produits tensoriels topologiques et espaces nucléaires, Memoirs Amer. Math. Soc. 16, 1955. MR0075539 (17:763c)

8. S. Kwapien, On a theorem of L. Schwartz and its applications to absolutely summing operators, Studia Math. 38 (1970), 193-201. MR0278090 (43:3822)

9. J. Lindenstrauss and L. Tzafriri, Classical Banach Spaces. I, Ergebnisse der Mathematik und ihrer Grenzgebiete 92, Springer-Verlag, Berlin-Heidelberg-New York, 1977. MR0500056 $(58: 17766)$

10. A. Persson and A. Pietsch, p-nukleare und p-integrale Abbildungen in Banachräumen, Studia Math. 33 (1969), 19-62. MR0243323 (39:4645)

11. A. Pietsch, Operator Ideals, North-Holland Publishing Company, Amsterdam-New YorkOxford, 1980. MR582655 (81j:47001)

12. C. Piñeiro, Sequences in the range of a vector measure of bounded variation, Proc. Amer. Math. Soc. 123 (1995), 3329-3334. MR1291790 (96a:46033)

13. C. Piñeiro and L. Rodríguez-Piazza, Banach spaces in which every compact lies inside the range of a vector measure, Proc. Amer. Math. Soc. 114 (1992), 507-517. MR 1086342 (92e:46038)

14. O. Reinov, A survey of some results in connection with Grothendieck approximation property, Math. Nachr. 119 (1984), 257-264. MR774195 (86d:46017)

15. D. P. Sinha and A. K. Karn, Compact operators whose adjoints factor through subspaces of $\ell_{p}$, Studia Math. 150 (2002), 17-33. MR.1893422 (2003g:46016) 
16. - Compact operators which factor through subspaces of $\ell_{p}$, Math. Nachr. 281 (2008), 412-423. MR2392123 (2009j:46037)

17. N. Tomczak-Jaegermann, Banach-Mazur Distances and Finite-Dimensional Operator Ideals, Pitman Monographs and Surveys in Pure and Applied Mathematics, 38, Longman Scientific \& Technical, Harlow; John Wiley \& Sons, New York, 1989. MR993774 (90k:46039)

Department of Mathematics (Faculty of Experimental Sciences), Campus Universitario de El Carmen, Avenida de las Fuerzas Armadas s/n, 21071 Huelva, Spain

E-mail address: candido@uhu.es

Department of Mathematics (Faculty of Experimental Sciences), Campus Universitario de El Carmen, Avenida de las Fuerzas Armadas s/n, 21071 Huelva, Spain

Current address: Departamento de Matemática Aplicada I (Escuela Técnica Superior de Arquitectura), Avenida de Reina Mercedes, 2, 41012 Sevilla, Spain

E-mail address: jmdelga@us.es 